\title{
Novel Therapeutic Targets of Tumor Metabolism
}

\author{
Rigel J. Kishton ${ }^{\star}{ }^{, a}$ and Jeffrey C. Rathmell ${ }^{\star}, t, \neq, b$ \\ *Department of Pharmacology and Cancer Biology, Duke University Medical Center, Durham, NC \\ 27710, USA \\ tDepartment of Immunology, Duke University Medical Center, Durham, NC 27710, USA \\ ‡Duke Molecular Physiology Institute, Duke University Medical Center, Durham, NC 27710, USA
}

\begin{abstract}
The study of tumor metabolism has resulted in new understandings of how cancer cells modify metabolic pathways that control cellular energetics to allow increased proliferation and survival. Tumor cells have been shown to alter metabolic pathways involved in glucose, glutamine and mitochondrial metabolism to generate raw materials needed for rapid cellular proliferation, maintain favorable cellular redox environments, modify cellular epigenetics and even promote and maintain oncogenic transformation. As a consequence, there has been intense scientific and clinical interest in targeting metabolic alterations that are commonly adopted by tumor cells for therapeutic purposes. In this review, we describe common metabolic alterations seen in tumor cells and discuss how these alterations are being investigated as potential targets for pharmacological intervention in preclinical and clinical settings. We also discuss some of the challenges associated with using tumor metabolism as a therapeutic target in cancer therapy, along with potential avenues to overcome these challenges.
\end{abstract}

\section{Keywords}

Tumor metabolism; aerobic glycolysis; glutamine; IDH mutations; combination therapy

\begin{abstract}
Therapeutic strategies for the treatment of cancer have undergone a revolution in recent years. In the past, frontline cancer therapy consisted largely of widely cytotoxic drugs that damaged both cancer cells and normal, healthy tissue. Over the past several decades, emphasis has been placed on identifying distinctive or preferential features of cancer cells to better target cancer yet spare normal cells. This has resulted in targeted therapies with higher treatment efficacy and improved patient outcomes. One recent focus in cancer research has been to exploit metabolic features of cancer cells that may separate them from normal tissue. The rationale behind this endeavor is that cancer cells, due to their rapid, sustained growth and proliferation and need to withstand hypoxia, must employ a metabolic program that
\end{abstract}

\footnotetext{
Address correspondence to: Jeffrey Rathmell, DUMC Box 3813, Duke University Medical Center, Durham, NC, 27710, Phone: 919-681-1084, Fax: 919-613-6606, jeff.rathmell@duke.edu.

aDoctoral candidate, MS

${ }^{\mathrm{b}}$ Associate Professor, $\mathrm{PhD}$

Conflicts of Interest: The authors declare no conflicts of interest.
} 
deviates from the metabolic characteristics of normal, healthy tissue. The ability to target cancer metabolism may result in a therapeutic window where cancer cells are inhibited but normal healthy cells remain unaffected.

\section{Metabolic Features of Cancer}

Researchers and clinicians working in cancer related fields have come to understand that cancer is a heterogeneous disease. There is tremendous variation in biology between different types of cancer, different patients with the same type of cancer, and even between different cancer cells in the same tumor. Cancer metabolism also exhibits considerable heterogeneity, with tumors adopting various metabolic programs that best suit a particular microenvironment. There are, however, some commonalities in cancer metabolism that are generally applicable to a significant portion of tumors. Among these common features of cancer metabolism are alterations in glucose, glutamine and mitochondrial metabolism. These commons features may allow for the development of novel therapeutics to target a fundamental hallmark of cancer biology. Indeed, the fundamental nature of some metabolic pathways may provide a unified target to bypass and overcome the genetic heterogeneity of tumors.

\section{Aerobic glycolysis in cancer}

The metabolic needs of most normal differentiated cells are largely energetic and metabolism is oriented towards maximal ATP generation through oxidative phosphorylation. During this process, glucose is first converted through glycolysis to pyruvate in the cytosol of the cell then further metabolized in the mitochondria in the TCA cycle and electron transport to produce an electromotive force that generates large quantities of ATP. Otto Warburg first noted nearly 100 years ago, however, that cancer cells use an altered program of glucose metabolism, in which they, even under normoxic conditions, convert glucose-derived pyruvate to lactate, rather than oxidizing it in the mitochondria[1]. This metabolic program differed from conventional models at the time, where lactate was thought produced only in anaerobic conditions, and instead resembles the Crabtree effect, wherein respiration is suppressed the presence of oxygen if glucose levels are sufficiently high. Warburg termed this metabolic trait aerobic glycolysis and hypothesized it resulted from mitochondrial defects in cancer cells that rendered them unable to utilize a normal, oxidative metabolic program. While it has since been found that most cancer cells have intact mitochondria, it is certainly true that a number of oncogenic driver mutations shift cell metabolism away from oxidative phosphorylation towards glycolytic metabolism[2, 3]. The teleological reason for this metabolic reprogramming remains uncertain, but a strong consensus has emerged that glycolytic use of glucose allows cancer cells to generate biosynthetic intermediates that are necessary for cell growth and proliferation, while also avoiding the production of potentially harmful reactive oxygen species (ROS) that results from oxidative phosphorylation. This view is buttressed by findings of similar metabolisms in proliferative normal healthy cells, such as lymphocytes and endothelial cells in angiogenesis. 
Since Warburg's description of aerobic glycolysis in cancer cells, key questions that have been studied are to what extent cancer cells require this metabolic program and can it be inhibited to eliminate cancer cells without excessive toxicity. There are a great number of metabolic pathways that utilize glucose or its derivatives towards the production of raw materials for biosynthesis, maintaining a favorable redox environment, and meeting the energy demands of cancer cells. Likewise amino acids can feed into a wide array of metabolic pathways. Given that core metabolic pathways are shared in nearly all cells, specificity for cancer cells and toxicity to normal cells are critical concerns. Also important is to what extent cancer cells can exert plasticity and respond to metabolic inhibition with compensatory metabolic reprogramming that may maintain cancer cell proliferation or viability.

\section{Targeting Aerobic Glycolysis}

There are numerous proteins that regulate the glycolytic pathway and have been proposed as potential drug targets. Here we will discuss the potential for targeting the early stages of glycolysis, where glucose is taken up into the cancer cell and phosphorylated to trap it in the cell, and the late stages of glycolysis, at the branch point where glucose derived pyruvate is either fluxed into the TCA cycle as acetyl-CoA, or converted to lactate for export (Figure1). The first two steps of glycolysis are the uptake of glucose into the cell by glucose transporters and subsequent phosphorylation by hexokinases. In numerous types of cancer, glucose transporters[4-6] and various isoforms of hexokinase are overexpressed[7], making them tempting targets for pharmacological inhibition. Indeed, the genetic deletion of Glut1 in a mouse model of B cell acute lymphoblastic leukemia (B-ALL) greatly slowed cell proliferation and lessened disease burden[8]. Similarly, the inhibition of glucose transporters has been explored in several cancer settings. For example, small molecule based inhibition of Glut1 was found to slow the growth of non-small cell lung cancer (NSCLC)[9] and have effects against renal cell carcinoma[3]. A number of drugs in the retroviral protease inhibitor class, commonly used to treat HIV infection, have been found to also possess the off-target effect of inhibiting glucose transporters, including Glut1 and Glut4[10]. Ritonavir, a drug in this class, has been shown to have anti-proliferative effects in a mouse model of multiple myeloma through the inhibition of glucose uptake into the cells[11]. When considering glucose transporters as a potential therapeutic target for human cancer patients, it must be noted that it is unclear what toxicities would occur with potent inhibition. For instance, Glut1 is heavily expressed at the blood brain barrier[12], and inhibition may result in neurological effects, as evidenced by patients with Glut1-deficiency Syndrome[13]. Nevertheless, Glut1 inhibitors with proven clinical track records, such as Ritonavir, show that a therapeutic window of partial inhibition of glucose uptake may be present.

Hexokinase may also provide a target in cancer metabolism through isoform selective inhibition. Several different types of cancer have been shown to overexpress Hexokinase II, an isoform not expressed in most normal tissue. Multiple groups have shown that the genetic deletion of Hexokinase II is beneficial, slowing cancer progression and reducing cancer cell survival in several different types of cancer, including lung, breast[14] and brain[15, 16]. Interestingly, while germline deletion of Hexokinase II is embryonic lethal in mice, whole body knockout in adult mice was reported well tolerated[14, 16], demonstrating that cancer 
cells may selectively rely on this isoform that could allow therapeutic targeting of Hexokinase II in cancer. Small molecules that broadly inhibit hexokinase, such as 2deoxyglucose (2-DG), have been shown to have activity against cancer in vitro[17-19] although in vivo efficacy of 2-DG as a single agent is modest $[8,20]$. However, these compounds are not specific for a particular hexokinase isoform, and continued development of small molecules targeted at hexokinase isoforms overexpressed in cancer may provide improved specificity.

An important early step in glycolysis is the conversion of fructose-6-phosphate to fructose-1,6-bisphosphate by 6-phosphofructo-1-kinase (PFK1). This is the first committed step in glycolysis, and the activity of PFK1 is elevated in many types of cancer, allowing for increased flux of glucose into the glycolytic pathway [21,22]. The mechanism of increased PFK1 activity in cancer relies upon the generation of an allosteric activator of PFK1. Oncogenic signaling increases the expression of an isoform of the 6-phosphofructo-2kinase/fructose-2,6-bisphosphatase (PFKFB) family of enzymes known as PFKFB3[23, 24]. Increased PFKFB3 expression results in the production of fructose-2,6,-bisphosphate, a potent allosteric activator of PFK1[25, 26]. Studies suggest that inhibition of PFKFB3 using genetic approaches[27] and small molecule inhibition[28] results in dramatically reduced glycolytic flux and slowed cancer cell growth. Early phase clinical trials are currently underway with small molecule PFKFB3 inhibitors [29].

Another key step in glucose metabolism is the branch point at which glycolysis-derived pyruvate can either be imported into the mitochondria to be oxidized in the TCA cycle, or converted to lactate in the cytosol. The pyruvate dehydrogenase (PDH) complex, which converts pyruvate to acetyl-CoA in the mitochondria, is responsible for regulating this key junction in pyruvate fate. An important regulator of PDH activity is pyruvate dehydrogenase kinase (PDHK). PDHK reduces the activity of PDH via inhibitory phosphorylation[30], resulting in decreased flux of pyruvate into the mitochondria, and increased production of lactate[31]. Several isoforms of PDHK have been shown to be overexpressed in various cancers [32-34], and play an important role in maintaining aerobic glycolysis in tumors. Numerous studies have shown that the inhibition of PDHK through RNAi or a small molecule inhibitor, dichloroacetate (DCA), caused cancer cell death in vitro and improved outcome in in vivo models of disease[35-37]. DCA was shown to alter the energetic balance of cancer cells, promoting the oxidation of glucose and consequent production of ROS[36-39]. DCA has been utilized clinically for the treatment of lactic acidosis[40], and several clinical trials have explored DCA as an anti-cancer treatment. In a small clinical trial, DCA treatment was associated with radiological regression of glioblastoma multiforme (GBM) in some patients, along with reduced proliferation and increased apoptosis of cancer cells[38]. Targeting PDHK with DCA or other novel small molecule inhibitors may be an effective strategy for the inhibition of aerobic glycolysis.

The lactate dehydrogenase (LDH) complex also plays a key role to regulate the fate of pyruvate in cancer. LDH is responsible for the conversion of pyruvate to lactate in the cytosol of the cell, and has increased expression and activity in a variety of cancer types[41, 42]. There are two isoforms of LDH that form tetramers of mixed composition[43] and increased presence of the LDHa isoform is often implicated in contributing to aerobic 
glycolysis in cancer cells[41, 42, 44]. Of the isoforms of LDH, LDHa has the highest affinity for pyruvate, along with the highest Vmax for enzymatic activity[45]. Thus, LDHa is able to rapidly convert pyruvate into lactate, completing aerobic glycolysis. There are several hypothesized reasons for cancer cells to overexpress LDHa and to convert pyruvate to lactate. The reaction catalyzed by LDHa results in the production of NAD+, which is critical for maintaining the activity of other proteins in the glycolytic pathway such as GAPDH[45, 46]. Also, studies have shown that LDHa activity is critical for keeping a favorable redox environment in cancer cells[47]. Several research groups have shown that the inhibition of LDHa by small molecule inhibitors or genetic approaches results in slowed cancer cell growth and increased cell death in a variety of types of cancer settings, including hepatocellular carcinoma and breast cancer[47-49]. There have been several early stage clinical trials to evaluate the efficacy of a non-specific inhibitor of $\mathrm{LDH}$, with mixed results observed[50, 51]. The pre-clinical development of inhibitors that have more specificity for LDHa is currently ongoing $[52,53]$.

\section{Glutamine metabolism in cancer}

In addition to altered glucose metabolism cancer cells can have increased usage of and reliance on glutamine for cell growth and survival. Dramatically increased usage of glutamine is a metabolic phenotype often associated with oncogenic Myc signaling[54], but is also found in tumors with other driver mutations such as oncogenic KRAS[55]. Glutamine serves several purposes to tumor cells (Figure 2). The first step in glutamine metabolism is its import into the cell via glutamine transporters. There are several known transporters that are capable of taking up glutamine, with SLC1A5 (ASCT2) and LAT1 being commonly upregulated in malignancies[56-58]. Once cytosolic, glutamine can be used as a substrate for the de novo synthesis of proteins, purines and pyrimidines[59], or can be converted to glutamate by enzymes called glutaminases (GLS). After conversion, glutamine derived glutamate can then be utilized by cancer cells for a variety of important purposes[60, 61]. One of these is the generation of non-essential amino acids for growth and proliferation through transamination of glutamate. Glutamate also plays an important role as a carbon donor in cancer cell TCA flux. Glutamate can be converted to a-ketoglutarate by glutamate dehydrogenase (GDH) and then fluxed into the TCA cycle where it can be used to support oxidative phosphorylation, the production of lipids, or to replenish key intermediates such as oxaloacetate[62, 63]. Glutamate can also be used to produce reducing agents for the cell, either being converted into glutathione[64], or generating NADPH through malic enzyme[65].

\section{Targeting glutamine metabolism}

The increased reliance by some cancers on glutamine metabolism provides several targets for therapeutic intervention. Several groups have explored the inhibition of glutamine transporters to limit glutamine uptake into cancer cells. LAT1 is inhibited by the small molecule inhibitor 2-amino-(2,2,1)-heptane-2-carboxylic acid (BCH), and the treatment of cancer cells in vitro and in vivo with BCH or genetic knockdown of LAT1 has been shown to slow proliferation and tumor growth[66, 67]. The inhibition of ASCT2 by RNAi or the small molecule L- $\gamma$-glutamyl-p-nitroanilide (GPNA) has also been shown to decrease pro- 
growth mTOR signaling and to induce autophagy in cancer cells[68]. Another study found that ASCT2 inhibition caused reduced growth and viability in several subtypes of lung cancer cells, effects that were mediated through a reduction in mTOR pathway activity [69]. Another potential therapeutic target in glutamine metabolism is GLS. A number of small molecule inhibitors of GLS have been developed, among them bis-2-(5phenylacetamido-1,2,4-thiodiazol-2-yl)ethyl sulfide (BPTES). BPTES has been shown to successfully inhibit GLS activity in several cancer settings, resulting in slowed growth and cell death $[70,71]$. There has also been interest in limiting the process of glutamine entering the TCA cycle as a-ketoglutarate by inhibiting GDH. Currently, there are no small molecule inhibitors that are specific for GDH[61]. However, non-specific inhibitors of GDH such as epigallocatechin gallate (EGCG) and aminooxyacetate (AOA) have been successfully demonstrated to be toxic to cancer cells in vitro, and to slow the growth of xenografted tumors[72, 73]. The development of more specific inhibitors of GDH may allow for more efficacious targeting of glutamine flux into the TCA cycle.

\section{Alterations to the TCA cycle in cancer metabolism}

In addition to utilizing aerobic glycolysis and glutamine metabolism for proliferation and survival, it has become clear in recent years that some cancer cells extensively also alter normal TCA cycle metabolism towards these ends (Figure 3). Typically thought of as acting in support of oxidative mitochondrial metabolism, the TCA cycle can also be adapted to produce cell building blocks for proliferation. As one example, citrate produced from acetyl CoA in the TCA cycle can be exported from the mitochondria and converted into raw material for the synthesis of fatty acids that are needed for cell proliferation. The TCA cycle flow can be reversed in reductive carboxylation so that a-ketoglutarate is converted to isocitrate then citrate for lipid synthesis[74, 75]. Also, interestingly, mutations that contribute to oncogenesis and the maintenance and progression of established tumors have been identified in several TCA enzymes. To date, several mutations have been identified in TCA cycle enzymes, including succinate dehydrogenase (SDH), fumarate hydratase (FH) and isocitrate dehydrogenase (IDH). SDH and FH have come to be thought of as tumor suppressors, as mutations in either enzyme has been shown to cause sarcomas, renal cell carcinoma and other rare types of cancer[76-78]. IDH mutations are found in gliomas[79, 80] and acute myeloid leukemias[81], and evidence implicates them in other cancer settings[82-85]. These mutations result in a gain of function, allowing IDH to begin producing a new "oncometabolite" called (R)-2-hydroxyglutarate (2HG)[86, 87]. 2HG itself has been termed an oncometabolite and has the capacity to transform immortalized cells in vitro [88, 89], through mechanisms that remain somewhat unclear. Numerous studies provide evidence that increased $2 \mathrm{HG}$ in cells harboring IDH mutations can inhibit demethylases, leading to hypermethylated DNA and retention of a stem cell-like phenotype[89-92].

\section{Targeting the TCA cycle in cancer metabolism}

Small molecule based targeting of abnormalities in the TCA cycle has become one of the greatest successes to date in therapeutically attacking cancer metabolism. While success targeting mutant $\mathrm{FH}$ and SDH with small molecule inhibitors has been limited because these are loss of function mutations, novel compounds that inhibit the gain-of-function activity of 
mutant IDH have recently been shown to have success in preclinical and clinical settings. In preclinical studies, small molecule inhibition of mutant IDH has been shown to dramatically reduce the production of $2 \mathrm{HG}$ and cause cancerous cells to differentiate towards a more normal phenotype[93, 94]. Early phase clinical trials have begun with a small molecule inhibitor of mutant IDH2, AG-221.

\section{Challenges of targeting cancer metabolism}

While there are numerous potential therapeutic targets in cancer metabolism, there are profound challenges associated with utilizing metabolic inhibition as a clinical strategy. First among these challenges is the fact that it is difficult to achieve a therapeutic window in cancer metabolism, as many normal cells, especially rapidly proliferating cells of the immune system, also utilize metabolic programs similar to those utilized by cancer and toxicity could be significant by targeting some metabolic pathways. For example, effector subtypes of T cells and antibody producing B cells also rely on aerobic glycolysis[95-97] and glutamine metabolism[98, 99] to maintain immune function. Metabolic inhibition of immune cells could potentially reduce their ability to fight cancer, and further, leave patients more vulnerable to opportunistic infections. Another challenge in targeting cancer metabolism is the metabolic flexibility that many tumor cells exhibit. Except for cases where there are actual mutations in metabolic genes, cancer cells often have a remarkable ability to shift fuel sources when deprived of favored metabolic pathways[8, 73, 100, 101]. This metabolic flexibility may limit the efficacy of targeting a single pathway for therapeutic purposes.

\section{Combination therapy as a solution}

One potential way to overcome the challenges posed to successfully utilize cancer metabolism as a therapeutic target is to utilize combination therapy. There are several potential ways in which metabolic combination therapy could be used against cancer cells. Inhibiting a primary metabolic pathway, followed by the subsequent inhibition of alternate metabolic pathways used by tumor cells might be one strategy. Additionally, many groups have shown that the partial inhibition of metabolic pathways utilized by cancer cells can dramatically sensitize the cancer cells to more traditional chemotherapeutic drugs or targeted therapies[8, 102-105]. This adjuvant metabolic sensitization may allow the use of far lower doses of both metabolic inhibitors and chemotherapeutic agents to achieve greater efficacy against tumor cells and reduced off-target effects.

\section{Conclusions}

The therapeutic potential of targeting the alterations of cellular metabolism in cancer has existed since the description of aerobic glycolysis by Otto Warburg. In the decades since Warburg's observation, much progress has been made in understanding exactly how many types of cancer alter cellular energetic pathways and how these alterations may be used to design novel therapeutic strategies to combat the disease. It is clear from recent research that there are a number of potential pathways and targets that may be beneficial targets for cancer therapy. In this review, we have described potential targets in the metabolic pathways 
that regulate glycolysis, glutamine metabolism, and the TCA cycle. It is likely that research in the coming years will identify more potential targets for therapeutic intervention. While there are numerous challenges associated with targeting cancer metabolism, among them off-target effects of metabolic inhibitors and the suppression of immune cells, strategies such as using metabolic inhibitors in combination therapy may allow for more effective clinical use.

\section{Acknowledgments}

The authors wish to thank members of the Rathmell lab for helpful discussions during the preparation of this manuscript. This work was supported by NIH R01CA123350 (JCR), NIH R03AI106835 (JCR), Leukemia and Lymphoma Society (JCR), NIH F31CA183529 (RJK), Duke Cancer Institute Pilot Grant (JCR).

\section{References}

1. Warburg O. On the origin of cancer cells. Science. 1956; 123(3191):309-314. [PubMed: 13298683]

2. DeBerardinis RJ, et al. The biology of cancer: metabolic reprogramming fuels cell growth and proliferation. Cell Metab. 2008; 7(1):11-20. [PubMed: 18177721]

3. Jones RG, Thompson CB. Tumor suppressors and cell metabolism: a recipe for cancer growth. Genes Dev. 2009; 23(5):537-48. [PubMed: 19270154]

4. Younes M, et al. Wide expression of the human erythrocyte glucose transporter Glut1 in human cancers. Cancer Res. 1996; 56(5):1164-7. [PubMed: 8640778]

5. Chan DA, et al. Targeting GLUT1 and the Warburg effect in renal cell carcinoma by chemical synthetic lethality. Sci Transl Med. 2011; 3(94):94ra70.

6. Flavahan WA, et al. Brain tumor initiating cells adapt to restricted nutrition through preferential glucose uptake. Nat Neurosci. 2013; 16(10):1373-82. [PubMed: 23995067]

7. Smith TA. Mammalian hexokinases and their abnormal expression in cancer. Br J Biomed Sci. 2000; 57(2):170-8. [PubMed: 10912295]

8. Liu T, et al. Glucose transporter 1-mediated glucose uptake is limiting for B-cell acute lymphoblastic leukemia anabolic metabolism and resistance to apoptosis. Cell Death Dis. 2014; 5:e1516.

9. Liu Y, et al. A small-molecule inhibitor of glucose transporter 1 downregulates glycolysis, induces cell-cycle arrest, and inhibits cancer cell growth in vitro and in vivo. Mol Cancer Ther. 2012; 11(8): 1672-82. [PubMed: 22689530]

10. Murata H, Hruz PW, Mueckler M. The mechanism of insulin resistance caused by HIV protease inhibitor therapy. J Biol Chem. 2000; 275(27):20251-4. [PubMed: 10806189]

11. McBrayer SK, et al. Multiple myeloma exhibits novel dependence on GLUT4, GLUT8, and GLUT11: implications for glucose transporter-directed therapy. Blood. 2012; 119(20):4686-97. [PubMed: 22452979]

12. Flier JS, et al. Distribution of glucose transporter messenger RNA transcripts in tissues of rat and man. J Clin Invest. 1987; 79(2):657-61. [PubMed: 3027132]

13. Klepper J, Voit T. Facilitated glucose transporter protein type 1 (GLUT1) deficiency syndrome: impaired glucose transport into brain-- a review. Eur J Pediatr. 2002; 161(6):295-304. [PubMed: 12029447]

14. Patra KC, et al. Hexokinase 2 is required for tumor initiation and maintenance and its systemic deletion is therapeutic in mouse models of cancer. Cancer Cell. 2013; 24(2):213-28. [PubMed: 23911236]

15. Wolf A, et al. Hexokinase 2 is a key mediator of aerobic glycolysis and promotes tumor growth in human glioblastoma multiforme. J Exp Med. 2011; 208(2):313-26. [PubMed: 21242296]

16. Gershon TR, et al. Hexokinase-2-mediated aerobic glycolysis is integral to cerebellar neurogenesis and pathogenesis of medulloblastoma. Cancer Metab. 2013; 1(1):2. [PubMed: 24280485] 
17. Ciavardelli D, et al. Breast cancer stem cells rely on fermentative glycolysis and are sensitive to 2deoxyglucose treatment. Cell Death Dis. 2014; 5:e1336. [PubMed: 25032859]

18. Zhang XD, et al. Effect of 2-deoxy-D-glucose on various malignant cell lines in vitro. Anticancer Res. 2006; 26(5A):3561-6. [PubMed: 17094483]

19. Coloff JL, et al. Akt-dependent glucose metabolism promotes Mcl-1 synthesis to maintain cell survival and resistance to Bcl-2 inhibition. Cancer Res. 2011; 71(15):5204-13. [PubMed: 21670080]

20. Maschek G, et al. 2-deoxy-D-glucose increases the efficacy of adriamycin and paclitaxel in human osteosarcoma and non-small cell lung cancers in vivo. Cancer Res. 2004; 64(1):31-4. [PubMed: 14729604]

21. Kole HK, et al. Regulation of 6-phosphofructo-1-kinase activity in ras-transformed rat-1 fibroblasts. Arch Biochem Biophys. 1991; 286(2):586-90. [PubMed: 1832835]

22. Hennipman A, et al. Glycolytic enzymes in breast cancer, benign breast disease and normal breast tissue. Tumour Biol. 1987; 8(5):251-63. [PubMed: 3448771]

23. Bobarykina AY, et al. Hypoxic regulation of PFKFB-3 and PFKFB-4 gene expression in gastric and pancreatic cancer cell lines and expression of PFKFB genes in gastric cancers. Acta Biochim Pol. 2006; 53(4):789-99. [PubMed: 17143338]

24. Atsumi T, et al. High expression of inducible 6-phosphofructo-2-kinase/fructose-2,6bisphosphatase (iPFK-2; PFKFB3) in human cancers. Cancer Res. 2002; 62(20):5881-7. [PubMed: 12384552]

25. Van Schaftingen E, Hue L, Hers HG. Fructose 2,6-bisphosphate, the probably structure of the glucose- and glucagon-sensitive stimulator of phosphofructokinase. Biochem J. 1980; 192(3):897901. [PubMed: 6453589]

26. Van Schaftingen E, et al. Control of liver 6-phosphofructokinase by fructose 2,6-bisphosphate and other effectors. Proc Natl Acad Sci U S A. 1981; 78(6):3483-6. [PubMed: 6455662]

27. Telang $S$, et al. Ras transformation requires metabolic control by 6-phosphofructo-2-kinase. Oncogene. 2006; 25(55):7225-34. [PubMed: 16715124]

28. Clem B, et al. Small-molecule inhibition of 6-phosphofructo-2-kinase activity suppresses glycolytic flux and tumor growth. Mol Cancer Ther. 2008; 7(1):110-20. [PubMed: 18202014]

29. Clem BF, et al. Targeting 6-phosphofructo-2-kinase (PFKFB3) as a therapeutic strategy against cancer. Mol Cancer Ther. 2013; 12(8):1461-70. [PubMed: 23674815]

30. Yeaman SJ, et al. Sites of phosphorylation on pyruvate dehydrogenase from bovine kidney and heart. Biochemistry. 1978; 17(12):2364-70. [PubMed: 678513]

31. Sugden MC, Holness MJ. Recent advances in mechanisms regulating glucose oxidation at the level of the pyruvate dehydrogenase complex by PDKs. Am J Physiol Endocrinol Metab. 2003; 284(5):E855-62. [PubMed: 12676647]

32. Hur H, et al. Expression of pyruvate dehydrogenase kinase-1 in gastric cancer as a potential therapeutic target. Int J Oncol. 2013; 42(1):44-54. [PubMed: 23135628]

33. Koukourakis MI, et al. Pyruvate dehydrogenase and pyruvate dehydrogenase kinase expression in non small cell lung cancer and tumor-associated stroma. Neoplasia. 2005; 7(1):1-6. [PubMed: 15736311]

34. McFate T, et al. Pyruvate dehydrogenase complex activity controls metabolic and malignant phenotype in cancer cells. J Biol Chem. 2008; 283(33):22700-8. [PubMed: 18541534]

35. Sanchez-Arago M, Chamorro M, Cuezva JM. Selection of cancer cells with repressed mitochondria triggers colon cancer progression. Carcinogenesis. 2010; 31(4):567-76. [PubMed: 20080835]

36. Bonnet $\mathrm{S}$, et al. A mitochondria-K+ channel axis is suppressed in cancer and its normalization promotes apoptosis and inhibits cancer growth. Cancer Cell. 2007; 11(1):37-51. [PubMed: 17222789]

37. Sutendra G, et al. Mitochondrial activation by inhibition of PDKII suppresses HIF1a signaling and angiogenesis in cancer. Oncogene. 2013; 32(13):1638-50. [PubMed: 22614004]

38. Michelakis ED, et al. Metabolic modulation of glioblastoma with dichloroacetate. Sci Transl Med. 2010; 2(31):31ra34.

Cancer J. Author manuscript; available in PMC 2016 March 01. 
39. Kaplon J, et al. A key role for mitochondrial gatekeeper pyruvate dehydrogenase in oncogeneinduced senescence. Nature. 2013; 498(7452):109-12. [PubMed: 23685455]

40. Stacpoole PW, Nagaraja NV, Hutson AD. Efficacy of dichloroacetate as a lactate-lowering drug. J Clin Pharmacol. 2003; 43(7):683-91. [PubMed: 12856382]

41. Koukourakis MI, et al. Lactate dehydrogenase 5 expression in squamous cell head and neck cancer relates to prognosis following radical or postoperative radiotherapy. Oncology. 2009; 77(5):28592. [PubMed: 19923867]

42. Koukourakis MI, et al. Lactate dehydrogenase 5 (LDH5) relates to up-regulated hypoxia inducible factor pathway and metastasis in colorectal cancer. Clin Exp Metastasis. 2005; 22(1):25-30. [PubMed: 16132575]

43. Markert CL, Shaklee JB, Whitt GS. Evolution of a gene. Multiple genes for LDH isozymes provide a model of the evolution of gene structure, function and regulation. Science. 1975; 189(4197):102-14. [PubMed: 1138367]

44. Koukourakis MI, et al. Lactate dehydrogenase-5 (LDH-5) overexpression in non-small-cell lung cancer tissues is linked to tumour hypoxia, angiogenic factor production and poor prognosis. $\mathrm{Br} \mathbf{J}$ Cancer. 2003; 89(5):877-85. [PubMed: 12942121]

45. Dawson DM, Goodfriend TL, Kaplan NO. Lactic Dehydrogenases: Functions of the Two Types Rates of Synthesis of the Two Major Forms Can Be Correlated with Metabolic Differentiation. Science. 1964; 143(3609):929-33. [PubMed: 14090142]

46. Doherty JR, Cleveland JL. Targeting lactate metabolism for cancer therapeutics. J Clin Invest. 2013; 123(9):3685-92. [PubMed: 23999443]

47. Le A, et al. Inhibition of lactate dehydrogenase A induces oxidative stress and inhibits tumor progression. Proc Natl Acad Sci U S A. 2010; 107(5):2037-42. [PubMed: 20133848]

48. Fantin VR, St-Pierre J, Leder P. Attenuation of LDH-A expression uncovers a link between glycolysis, mitochondrial physiology, and tumor maintenance. Cancer Cell. 2006; 9(6):425-34. [PubMed: 16766262]

49. Shim H, et al. c-Myc transactivation of LDH-A: implications for tumor metabolism and growth. Proc Natl Acad Sci U S A. 1997; 94(13):6658-63. [PubMed: 9192621]

50. Liu G, et al. An open-label, multicenter, phase I/II study of single-agent AT-101 in men with castrate-resistant prostate cancer. Clin Cancer Res. 2009; 15(9):3172-6. [PubMed: 19366825]

51. Baggstrom MQ, et al. A phase II study of AT-101 (Gossypol) in chemotherapy-sensitive recurrent extensive-stage small cell lung cancer. J Thorac Oncol. 2011; 6(10):1757-60. [PubMed: 21918390]

52. Manerba M, et al. Galloflavin (CAS 568-80-9): a novel inhibitor of lactate dehydrogenase. ChemMedChem. 2012; 7(2):311-7. [PubMed: 22052811]

53. Granchi C, et al. Discovery of N-hydroxyindole-based inhibitors of human lactate dehydrogenase isoform A (LDH-A) as starvation agents against cancer cells. J Med Chem. 2011; 54(6):1599-612. [PubMed: 21332213]

54. Gao P, et al. c-Myc suppression of miR-23a/b enhances mitochondrial glutaminase expression and glutamine metabolism. Nature. 2009; 458(7239):762-5. [PubMed: 19219026]

55. Son J, et al. Glutamine supports pancreatic cancer growth through a KRAS-regulated metabolic pathway. Nature. 2013; 496(7443):101-5. [PubMed: 23535601]

56. Fuchs BC, Bode BP. Amino acid transporters ASCT2 and LAT1 in cancer: partners in crime? Semin Cancer Biol. 2005; 15(4):254-66. [PubMed: 15916903]

57. Witte D, et al. Overexpression of the neutral amino acid transporter ASCT2 in human colorectal adenocarcinoma. Anticancer Res. 2002; 22(5):2555-7. [PubMed: 12529963]

58. Collins CL, et al. Determinants of glutamine dependence and utilization by normal and tumorderived breast cell lines. J Cell Physiol. 1998; 176(1):166-78. [PubMed: 9618156]

59. Cory JG, Cory AH. Critical roles of glutamine as nitrogen donors in purine and pyrimidine nucleotide synthesis: asparaginase treatment in childhood acute lymphoblastic leukemia. In Vivo. 2006; 20(5):587-9. [PubMed: 17091764]

60. Mates JM, et al. Glutaminase isoenzymes as key regulators in metabolic and oxidative stress against cancer. Curr Mol Med. 2013; 13(4):514-34. [PubMed: 22934847]

Cancer J. Author manuscript; available in PMC 2016 March 01. 
61. Hensley CT, Wasti AT, DeBerardinis RJ. Glutamine and cancer: cell biology, physiology, and clinical opportunities. J Clin Invest. 2013; 123(9):3678-84. [PubMed: 23999442]

62. Reitzer LJ, Wice BM, Kennell D. Evidence that glutamine, not sugar, is the major energy source for cultured HeLa cells. J Biol Chem. 1979; 254(8):2669-76. [PubMed: 429309]

63. Anderson ME, Meister A. Transport and direct utilization of gamma-glutamylcyst(e)ine for glutathione synthesis. Proc Natl Acad Sci U S A. 1983; 80(3):707-11. [PubMed: 6572362]

64. Meister A, Anderson ME. Glutathione. Annu Rev Biochem. 1983; 52:711-60. [PubMed: 6137189]

65. Lyssiotis CA, et al. Pancreatic cancers rely on a novel glutamine metabolism pathway to maintain redox balance. Cell Cycle. 2013; 12(13):1987-8. [PubMed: 23759579]

66. Kaira K, et al. Clinical significance of L-type amino acid transporter 1 expression as a prognostic marker and potential of new targeting therapy in biliary tract cancer. BMC Cancer. 2013; 13:482. [PubMed: 24131658]

67. Wang Q, et al. Targeting amino acid transport in metastatic castration-resistant prostate cancer: effects on cell cycle, cell growth, and tumor development. J Natl Cancer Inst. 2013; 105(19):146373. [PubMed: 24052624]

68. Nicklin P, et al. Bidirectional transport of amino acids regulates mTOR and autophagy. Cell. 2009; 136(3):521-34. [PubMed: 19203585]

69. Hassanein M, et al. SLC1A5 mediates glutamine transport required for lung cancer cell growth and survival. Clin Cancer Res. 2013; 19(3):560-70. [PubMed: 23213057]

70. Seltzer MJ, et al. Inhibition of glutaminase preferentially slows growth of glioma cells with mutant IDH1. Cancer Res. 2010; 70(22):8981-7. [PubMed: 21045145]

71. Emadi A, et al. Inhibition of glutaminase selectively suppresses the growth of primary acute myeloid leukemia cells with IDH mutations. Exp Hematol. 2014; 42(4):247-51. [PubMed: 24333121]

72. Qing G, et al. ATF4 regulates MYC-mediated neuroblastoma cell death upon glutamine deprivation. Cancer Cell. 2012; 22(5):631-44. [PubMed: 23153536]

73. Yang C, et al. Glioblastoma cells require glutamate dehydrogenase to survive impairments of glucose metabolism or Akt signaling. Cancer Res. 2009; 69(20):7986-93. [PubMed: 19826036]

74. Filipp FV, et al. Reverse TCA cycle flux through isocitrate dehydrogenases 1 and 2 is required for lipogenesis in hypoxic melanoma cells. Pigment Cell Melanoma Res. 2012; 25(3):375-83. [PubMed: 22360810]

75. Scott DA, et al. Comparative metabolic flux profiling of melanoma cell lines: beyond the Warburg effect. J Biol Chem. 2011; 286(49):42626-34. [PubMed: 21998308]

76. Pollard PJ, Wortham NC, Tomlinson IP. The TCA cycle and tumorigenesis: the examples of fumarate hydratase and succinate dehydrogenase. Ann Med. 2003; 35(8):632-9. [PubMed: 14708972]

77. Gottlieb E, Tomlinson IP. Mitochondrial tumour suppressors: a genetic and biochemical update. Nat Rev Cancer. 2005; 5(11):857-66. [PubMed: 16327764]

78. Raimundo N, Baysal BE, Shadel GS. Revisiting the TCA cycle: signaling to tumor formation. Trends Mol Med. 2011; 17(11):641-9. [PubMed: 21764377]

79. Balss J, et al. Analysis of the IDH1 codon 132 mutation in brain tumors. Acta Neuropathol. 2008; 116(6):597-602. [PubMed: 18985363]

80. Bleeker FE, et al. IDH1 mutations at residue p.R132 (IDH1(R132)) occur frequently in high-grade gliomas but not in other solid tumors. Hum Mutat. 2009; 30(1):7-11. [PubMed: 19117336]

81. Rakheja D, et al. IDH mutations in acute myeloid leukemia. Hum Pathol. 2012; 43(10):1541-51. [PubMed: 22917530]

82. Terunuma A, et al. MYC-driven accumulation of 2-hydroxyglutarate is associated with breast cancer prognosis. J Clin Invest. 2014; 124(1):398-412. [PubMed: 24316975]

83. Amary MF, et al. IDH1 and IDH2 mutations are frequent events in central chondrosarcoma and central and periosteal chondromas but not in other mesenchymal tumours. J Pathol. 2011; 224(3): 334-43. [PubMed: 21598255]

84. Amary MF, et al. Ollier disease and Maffucci syndrome are caused by somatic mosaic mutations of IDH1 and IDH2. Nat Genet. 2011; 43(12):1262-5. [PubMed: 22057236] 
85. Borger DR, et al. Frequent mutation of isocitrate dehydrogenase (IDH) 1 and IDH2 in cholangiocarcinoma identified through broad-based tumor genotyping. Oncologist. 2012; 17(1): 72-9. [PubMed: 22180306]

86. Dang L, et al. Cancer-associated IDH1 mutations produce 2-hydroxyglutarate. Nature. 2009; 462(7274):739-44. [PubMed: 19935646]

87. Ward PS, et al. The common feature of leukemia-associated IDH1 and IDH2 mutations is a neomorphic enzyme activity converting alpha-ketoglutarate to 2-hydroxyglutarate. Cancer Cell. 2010; 17(3):225-34. [PubMed: 20171147]

88. Losman JA, et al. (R)-2-hydroxyglutarate is sufficient to promote leukemogenesis and its effects are reversible. Science. 2013; 339(6127):1621-5. [PubMed: 23393090]

89. Lu C, et al. IDH mutation impairs histone demethylation and results in a block to cell differentiation. Nature. 2012; 483(7390):474-8. [PubMed: 22343901]

90. Chowdhury R, et al. The oncometabolite 2-hydroxyglutarate inhibits histone lysine demethylases. EMBO Rep. 2011; 12(5):463-9. [PubMed: 21460794]

91. Figueroa ME, et al. Leukemic IDH1 and IDH2 mutations result in a hypermethylation phenotype, disrupt TET2 function, and impair hematopoietic differentiation. Cancer Cell. 2010; 18(6):553-67. [PubMed: 21130701]

92. Turcan S, et al. IDH1 mutation is sufficient to establish the glioma hypermethylator phenotype. Nature. 2012; 483(7390):479-83. [PubMed: 22343889]

93. Rohle D, et al. An inhibitor of mutant IDH1 delays growth and promotes differentiation of glioma cells. Science. 2013; 340(6132):626-30. [PubMed: 23558169]

94. Wang F, et al. Targeted inhibition of mutant IDH2 in leukemia cells induces cellular differentiation. Science. 2013; 340(6132):622-6. [PubMed: 23558173]

95. Macintyre AN, et al. The glucose transporter Glut1 is selectively essential for CD4 T cell activation and effector function. Cell Metab. 2014; 20(1):61-72. [PubMed: 24930970]

96. Michalek RD, et al. Cutting edge: distinct glycolytic and lipid oxidative metabolic programs are essential for effector and regulatory CD4+ T cell subsets. J Immunol. 2011; 186(6):3299-303. [PubMed: 21317389]

97. Caro-Maldonado A, et al. Metabolic reprogramming is required for antibody production that is suppressed in anergic but exaggerated in chronically BAFF-exposed B cells. J Immunol. 2014; 192(8):3626-36. [PubMed: 24616478]

98. Le A, et al. Glucose-independent glutamine metabolism via TCA cycling for proliferation and survival in B cells. Cell Metab. 2012; 15(1):110-21. [PubMed: 22225880]

99. Nakaya M, et al. Inflammatory T cell responses rely on amino acid transporter ASCT2 facilitation of glutamine uptake and mTORC1 kinase activation. Immunity. 2014; 40(5):692-705. [PubMed: 24792914]

100. Choo AY, et al. Glucose addiction of TSC null cells is caused by failed mTORC1-dependent balancing of metabolic demand with supply. Mol Cell. 2010; 38(4):487-99. [PubMed: 20513425]

101. Chen V, Shtivelman E. CC3/TIP30 regulates metabolic adaptation of tumor cells to glucose limitation. Cell Cycle. 2010; 9(24):4941-53. [PubMed: 21150275]

102. Samudio I, et al. Pharmacologic inhibition of fatty acid oxidation sensitizes human leukemia cells to apoptosis induction. J Clin Invest. 2010; 120(1):142-56. [PubMed: 20038799]

103. Zhou M, et al. Warburg effect in chemosensitivity: targeting lactate dehydrogenase-A resensitizes taxol-resistant cancer cells to taxol. Mol Cancer. 2010; 9:33. [PubMed: 20144215]

104. Ayyanathan K, et al. Combination of sulindac and dichloroacetate kills cancer cells via oxidative damage. PLoS One. 2012; 7(7):e39949. [PubMed: 22866174]

105. Giacobbe A, et al. p63 regulates glutaminase 2 expression. Cell Cycle. 2013; 12(9):1395-405. [PubMed: 23574722] 


\section{Glossary}

2-DG

2HG

AOA

ATP

B-ALL

BCH

BPTES

DCA

EGCG

FH

GAPDH

GBM

GDH

GLS

GPNA

IDH

LAT1

LDH

mTOR

NAD+

NADPH

NSCLC

PDH

PDHK

PFK1

PFKFB

RNAi

ROS

SDH

SLC1A5 (ASCT2)

TCA cycle 2-deoxyglucose

2-hydroxyglutarate

aminoxyacetate

adenosine triphosphate

B cell acute lymphoblastic leukemia

2-amino-(2,2,1)-heptane-2-carboxylic acid

bis-2-(5-phenylacetamido-1,2,4-thiodiazol-2-yl)ethyl sulfide

dichloroacetate

epigallocatechin gallate

fumarate hydratase

glyceraldehyde 3-phosphate dehydrogenase

glioblastoma multiforme

glutamate dehydrogenase

glutaminase

L- $\gamma$-glutamyl-p-nitroanilide

isocitrate dehydrogenase

large neutral amino acid transporter

lactate dehydrogenase

mechanistic target of rapamycin

nicotinamide adenine dinucleotide

nicotinamide adenine dinucleotide phosphate

non-small cell lung cancer

pyruvate dehydrogenase

pyruvate dehydrogenase kinase

phosphofructokinase 1

6-phosphofructo-2-kinase/fructose-2,6-bisphosphatase

RNA interference

reactive oxygen species

succinate dehydrogenase

neutral amino acid transporter $\mathrm{B}(0) / \mathrm{ASC}$ amino acid transporter 2

tricarboxylic acid cycle 


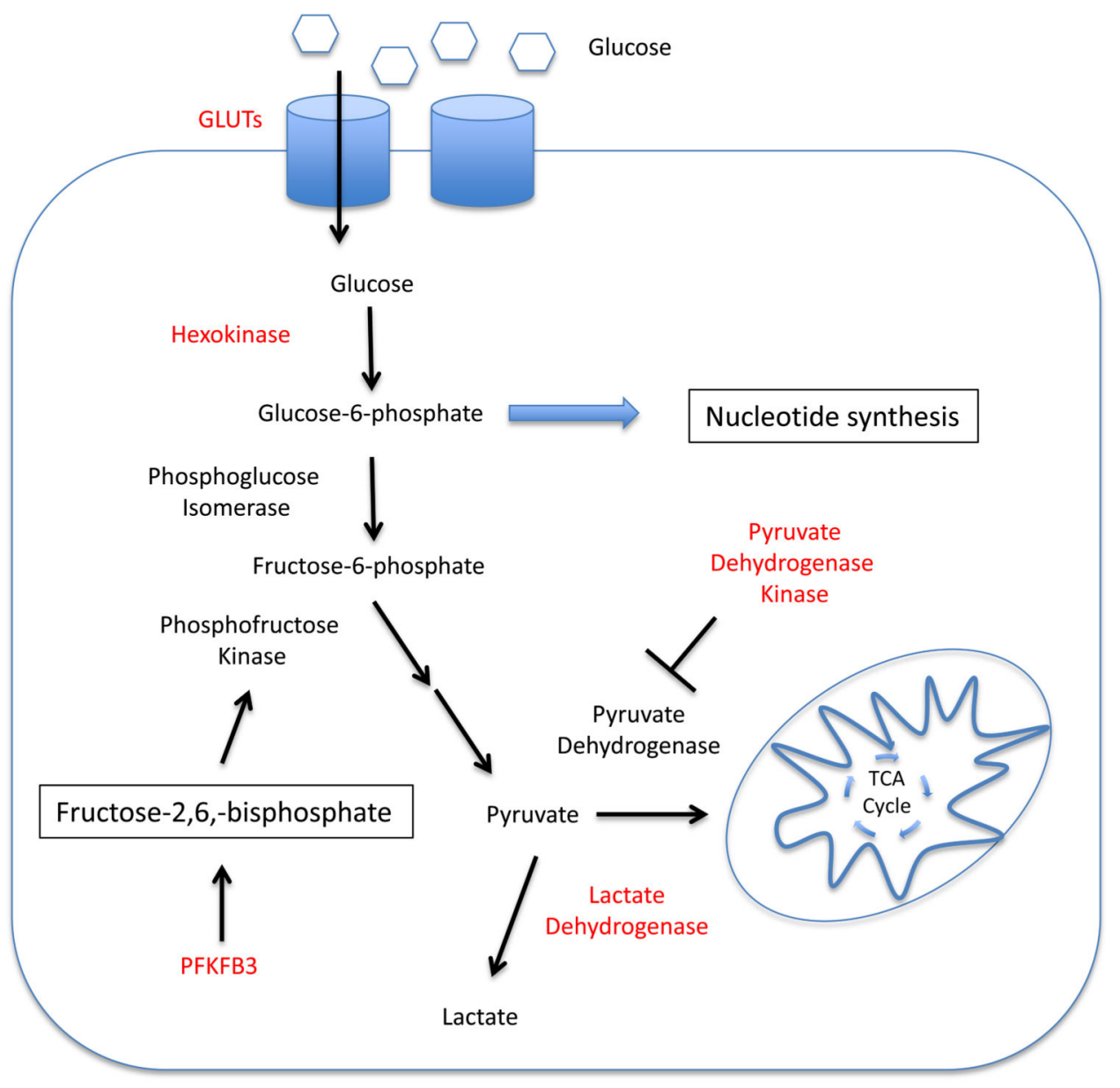

Figure 1. Tumor cell glucose metabolism as a therapeutic target

The alterations to normal glucose metabolism that are exhibited in cancer cells may provide targets for therapeutic intervention. Inhibiting aerobic glycolysis in cancer cells causes slowed cell proliferation and increased cell death. Enzymes that are currently being targeted with small molecule inhibitors are indicated in red. 


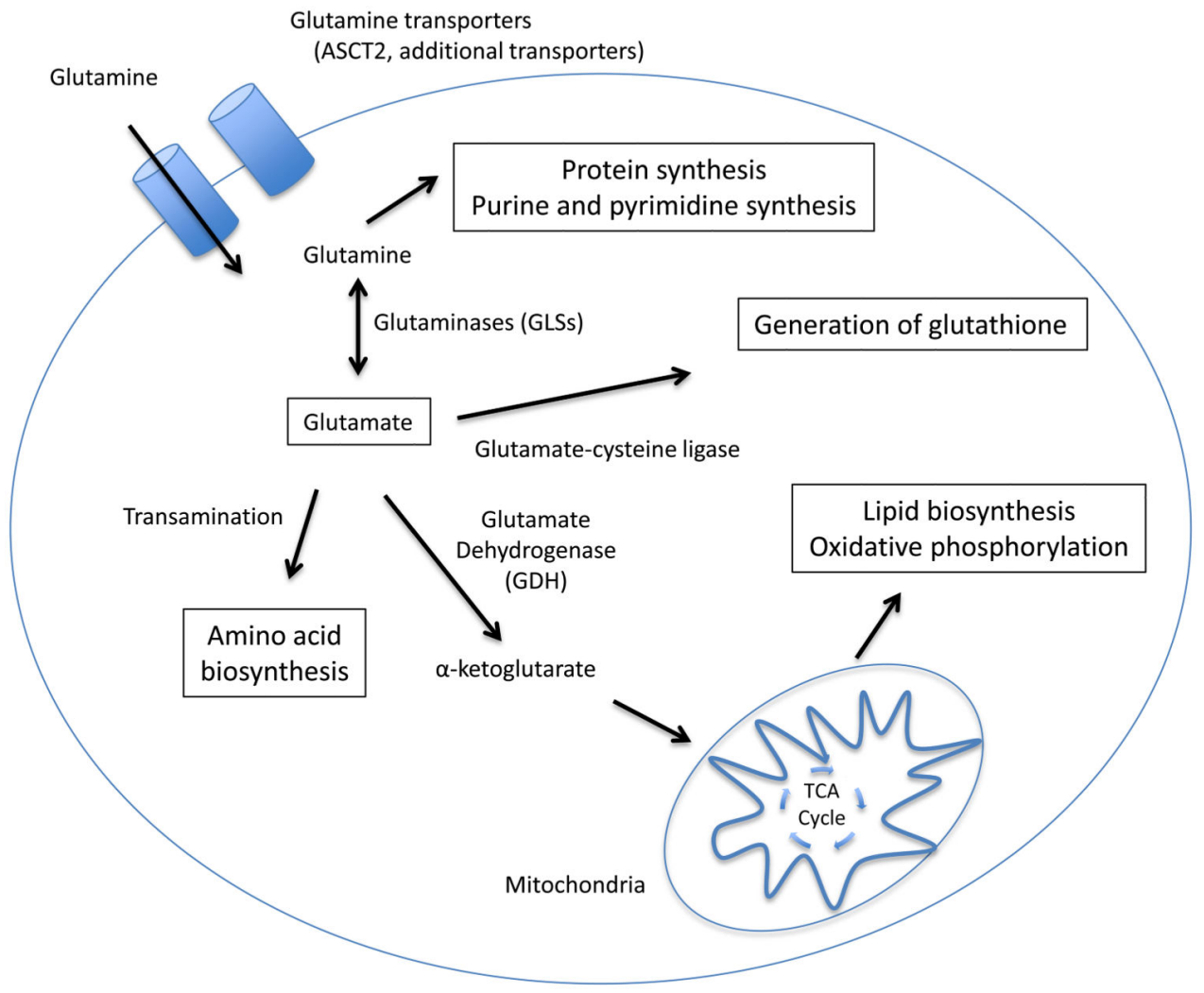

Figure 2. Glutamine is used for a number of anabolic processes by cancer cells Glutamine is transported inside tumor cells by glutamine transporters such as ASCT2. Once inside the cell, it can be used directly as a substrate for protein and nucleotide synthesis, or converted to glutamate by glutaminases (GLS). Glutamine-derived glutamate has a number of uses for cancer cells. It may be used to generate amino acids via transamination, or used in the generation of reducing equivalents such as glutathione. Additionally, glutamate may be converted to a-ketoglutarate by glutamate dehydrogenase $(\mathrm{GDH})$ and fluxed into the mitochondrial TCA cycle where it can be used to support oxidative phosphorylation, or used to generate lipids. 


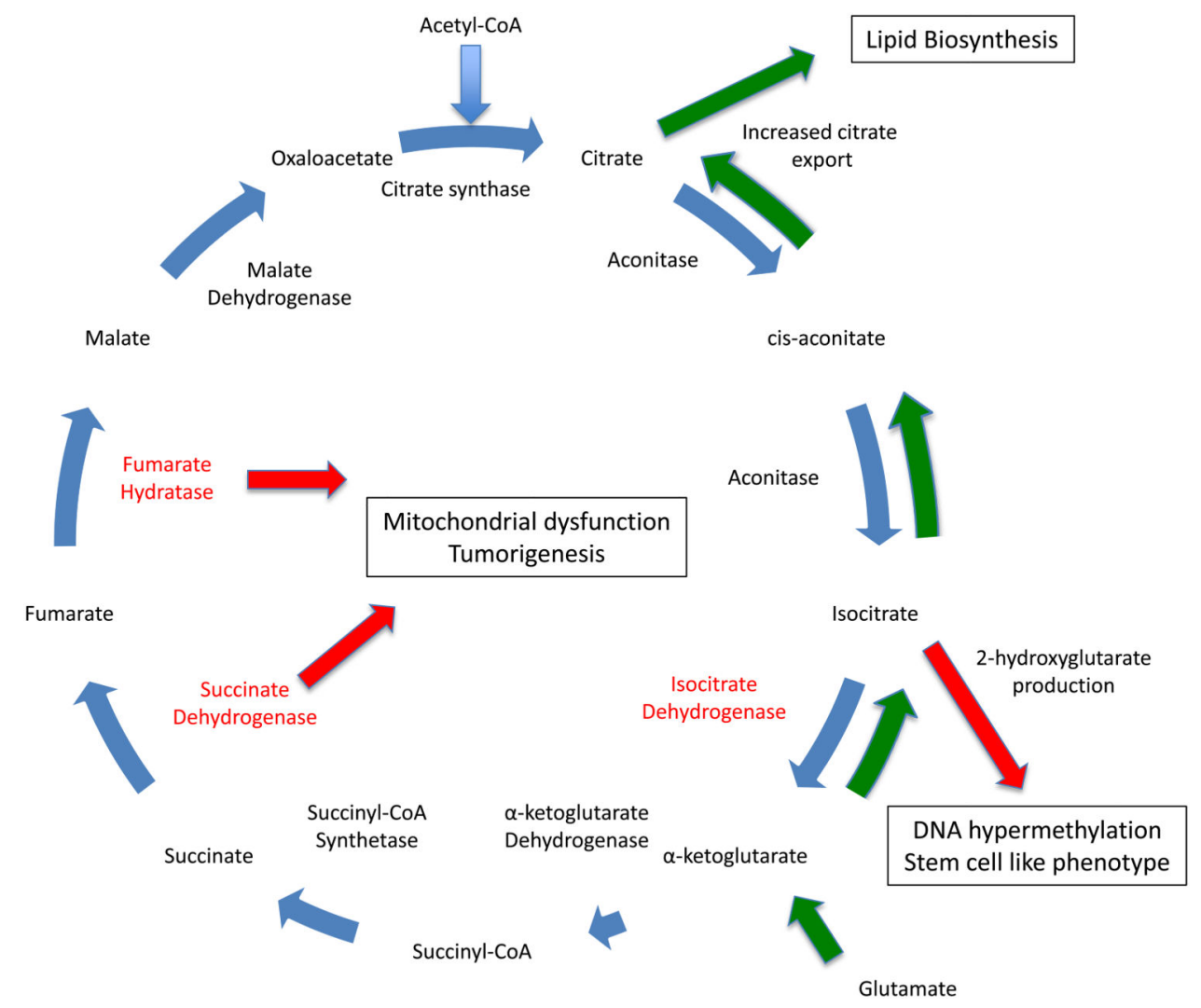

Figure 3. Cancer cells alter the TCA cycle to support proliferation and oncogenic transformation Tumor cells often significantly alter the flow of TCA cycle intermediates (common alterations indicated with green arrows) to increase the generation of substrates useful for cell growth and proliferation. One common alteration in TCA cycle flux is the increased export of citrate from the TCA cycle to support de novo lipid synthesis for proliferation. Along with simply increasing the amount of citrate that is exported, some cancer cells also utilize glutamine-derived glutamate to generate citrate. In this process, glutamate is converted to a-ketoglutarate and fluxed into the TCA cycle. The TCA cycle flow is then reversed, with a-ketoglutarate being converted into isocitrate and eventually citrate to yield even more substrate for lipid synthesis. Tumor cells also are known to have mutations in key TCA cycle enzymes (enzymes known to be mutated indicated in red). Isocitrate dehydrogenase mutations can result in the generation of the "oncometabolite" 2hydroxyglutarate, which contributes the a stem cell like phenotype in tumor cells. Additional mutations have been identified in fumarate hydratase and succinate dehydrogenase. These mutations result in mitochondrial dysfunction and contribute to oncogenic transformation. 